\title{
*A REPERCUSSÃO DE ARTIGOS DE PERIÓDICOS BRASILEIROS DE CIÊNCIA DA INFORMAÇÃO NO FACEBOOK: UM ESTUDO ALTMÉTRICO
}

THE PERFORMANCE IMPACT OF THE BRAZILIAN INFORMATION SCIENCE JOURNALS ON FACEBOOK: AN ALTMETRIC STUDY

LA REPERCUSIÓN DE ARTÍCULOS DE PERIÓDICOS BRASILEÑOS DE LA CIENCIA DE LA INFORMACIÓN EN FACEBOOK: UN ESTUDIO ALTMÉTRICO

${ }^{1}$ Ronaldo Ferreira Araujo

2Tiago Rodrigo Marçal Murakami

3Jorge Moisés Kroll do Prado

Universidade Federal de Alagoas ${ }^{1}$

Universidade de São Paulo ${ }^{2}$

Rede de Bibliotecas do Senac ${ }^{3}$

\author{
Correspondência \\ ${ }^{1}$ Ronaldo Ferreira Araujo \\ Universidade Federal de Alagoas \\ Maceió, AL - Brasil \\ Email: ronaldfa@gmail.com \\ ORCID: http://orcid.org/0000-0003-0778-9561
}

Submetido em: 29/09/2017

Aceito em: 18/12/2017

Publicado em: 19/01/2018

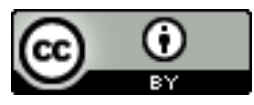

JITA: HT. Web 2.0, Social networks

*Comunicação apresentada originalmente no evento ABEC Meeting 2017 
RESUMO: Reflete sobre as interações do Facebook enquanto elementos de atenção online e fonte de dados altmétricos, e dialoga as reflexões com uma análise empírica que considerou a repercussão de artigos de periódicos da Ciência da Informação indexados no Repertório da Produção Periódica Brasileira de Ciência da Informação (RPPBCI). A coleta de dados altmétricos foi realizada por meio da utilização de Application Program Interface do Facebook parametrizada pela URL principal e secundária de artigos de 31 periódicos. A repercussão dos artigos indica uma atenção online com 13.633 interações, sendo 8.840 de "curtidas", 2.992 de "compartilhamentos" e 1.801 de "comentários". A revista Perspectivas em Ciência da informação possui o maior destaque com $13,4 \%$ da atenção recebida e artigos recentes obtiveram pontuação mais elevada. Concluise que interações com conteúdo de artigos científicos no Facebook são ricas fontes de dados altmétricos e merecem maior atenção por parte de estudiosos desse campo, elas podem ser benéficas como uma métrica agregada porque fornece uma visão adicional sobre o desempenho científico dos artigos quando curtidos, compartilhados ou comentados.

PALAVRAS-ChaVE: Altmetria. Atenção Online. Facebook.

ABSTRACT: This article reflects on the Facebook's interactions as elements of online attention and altmetric data. The empirical analysis of the reflections considers the repercussion of Information Science journal articles, indexed in the Periodical Brazilian Information Science Production Repertory (RPPBCI). The collection of the altmetric data was performed through Facebook's Application Program Interface parameterized by the main and secondary URL of articles from 31 journals. The articles' repercussion indicates an online attention with 13.633 interactions, of which 8.840 are "likes", 2.992 are "shares" and 1.801 are "comments". The Perspectivas em Ciência da Informação Journal has the greatest visibility with $13.4 \%$ of the attention received and recent articles achieved high score. In conclusion, interactions with the content of scientific articles on Facebook are rich sources of altmetric data and deserve more attention from scholars in this field. They can be helpful as an aggregate metric for providing an additional view of the articles' scientific performance when liked, shared or commented.

KEYWORDS: Altmetrics. Online attention. Facebook.

RESUMEN: Refleja sobre las interaciones del Facebook mientra elementos de atención en línea y fuente de datos altmétricos, y dialoga las reflexiones con un análisis empírico que consideró la repercusión de artículos de periódicos de la Ciencia de la Información indexados en el Repertorio de la Producción Periódica Brasileña de Ciencia de la Información (RPPBCI). La recolección de datos altmétricos fue realizada por medio de la utilización de Application Program Interface del Facebook parametrizada por la URL principal y secundaria de artículos de 31 periódicos. La repercusión de los artículos indica una atención en línea con 13.633 interacciones, siendo 8.840 "me gusta", 2.992 veces se ha compartido y 1.801 comentarios. La revista Perspectivas em Ciência da Informação posee el mayor destaque con el 13,4\% de la atención recibida y artículos recientes obtuvieron puntuación más elevada. Se concluye que las interaciones con contenido de artículos científicos en Facebook son ricas fuentes de datos altmétricos y merecen mayor atención por parte de estudiosos de ese campo, ellas pueden ser benéficas como una métrica agregada porque proporciona una visión adicional sobre el desempeño científico de los artículos cuando son curtidos, compartidos o comentados.

PALABRAS CLAVE: Altimetría. Atención en línea. Facebook. 


\section{INTRODUÇÃ̃o}

A apreciação e avaliação do impacto de publicações de resultados de pesquisa são processos fundamentais à ciência e servem ao avanço do conhecimento em nossa sociedade. Durante um bom tempo tais processos têm sido suportados por estudos métricos tradicionais de avaliações de desempenho com base em indicadores quantitativos específicos (ex. produtividade científica, número de citações e fatores de impacto) que trazem consigo inúmeras críticas, sobretudo devido à sua negligência dos aspectos qualitativos do desempenho científico.

O aumento cada vez maior do volume de produção científica refletido na quantidade de teses, dissertações, trabalhos apresentados em eventos e especial de artigos de periódicos tendo em vista a criação de novas revistas, bem como o movimento de mudança na frequência da periodicidade de publicação (BLATTMANN; SANTOS, 2014) - requer uma abordagem diferente, com processamento mais rápido e principalmente uma indicação mais clara de artigos relevantes.

As citações que artigos de periódicos recebem se consolidaram como bons indicadores de impacto, mas sua função é retrospectiva, uma vez que, as citações costumam acumular muito lentamente, não sendo adequadas para identificar artigos com assuntos "do momento", relevantes para ler e trabalhar ou mesmo, identificar temas de investigação mais populares (PRIEM; GROTH; TARABORELLI, 2012; BOON; FOON, 2014).

Além disso, as análises sobre o impacto do trabalho científico precisam se estender para além do discurso científico formal (SHEMA; BAR-ILAN; THELWALL, 2014) com vistas a atingir um público dentro e fora da comunidade científica (ARAUJO, 2015a; VANTI; SANZ-CASADO, 2016) e os blogs e mídias sociais como Facebook e Twitter têm desempenhado um importante papel nessa questão, no âmbito dos estudos das altmetrias ou métricas alternativas (GOUVEIA, 2013; BARROS, 2015).

Embora os indicadores altmétricos possam ser gerados por inúmeras fontes de mídias sociais, as quais possuem propósitos de usos distintos, parece haver uma predileção da comunidade científica pelo uso do Twitter em estudos que analisam o impacto social de pesquisas na web social (THELWALL et al., 2013; HAUSTEIN et al., 2014; HOLMBERG et al., 2014; WINTER, 2015; SCHNITZLER et al., 2016), deixando uma lacuna para estudos que reflitam sobre esse mesmo impacto em outras mídias, sobretudo considerando as contribuições dessas mídias e suas particularidades de uso para a comunicação científica.

Para essa necessária tarefa de refletir e aprofundar o conhecimento sobre o campo das métricas alternativas Gouveia (2016, p.644) reforça que devemos sim considerá-las, mas sem perder de vista "as particularidades que cada fonte de dados tem, e com suas influências na produção de atenção e interação de seus atores". 
Assim, o presente estudo tem por objetivo refletir sobre as interações do Facebook enquanto elementos de atenção online e fonte de dados altmétricos, e dialoga as reflexões com uma análise empírica que considerou 31 periódicos da Ciência da Informação cujos artigos estão indexados no Repertório da Produção Periódica Brasileira de Ciência da Informação (RPPB CI): <http://www.labimetrics.inf.br/rppbci/>. A pesquisa tem sido elaborada no Laboratório de Estudos Métricos da Informação na Web (Lab.iMetrics): < http://dgp.cnpq.br/dgp/espelho grupo/5207019450670905> na linha de pesquisa "Ciência 2.0 e os aportes da altmetria".

\section{O FACEBOOK COMO FONTE DE DADOS ALTMÉTRICOS}

O número crescente de usuários em sites de redes sociais e o aumento no tempo gasto conectado nesses ambientes têm feito alguns estudiosos refletirem sobre o uso dessas mídias como fonte e canal de informação, e inclusive, sobre a necessidade de incluí-las como filtro de informações científicas (BARROS, 2015). O Facebook é maior rede social do mundo, em 2015 já registrava pouco mais de 1,19 bilhões de usuários (BARTH; PACHECO; PINHEIRO, 2015) e tem grande potencial para desempenhar esse papel como filtro de informação.

Além de filtro de informação o Facebook também pode ser utilizado pelos periódicos científicos como mecanismo de divulgação dos artigos que publicam e popularização do conhecimento científico (SOUZA et al., 2015), bem como para reter e atrair leitores, autores e avaliadores, o que consequentemente, amplia sua rede de relacionamento (COSTA et al., 2016).

Para Allen (2016) o aumento do alcance que determinadas pesquisas atingem no Facebook demonstra o interesse público e o engajamento que as mesmas obtêm na rede. Para a autora, medir o alcance de seus trabalhos nesta mídia social possibilita aos pesquisadores compreender melhor a repercussão que eles recebem.

$\mathrm{Na}$ esfera conversacional as interações ou ações de curtir, compartilhar e comentar no Facebook são vistas como apropriações simbólicas e podem indicar intenções distintas. Para Recuero (2014) o curtir indicaria uma ação de: a) difusão da informação, uma vez que o usuário ao fazê-lo torna público a toda a sua rede social a mensagem "curtida"; e b) difusão de apoio e concordância, funcionando como uma forma de legitimação do conteúdo "curtido".

O compartilhar tem outro papel e valores associados e sua principal função parece ser a de "dar visibilidade para a conversação ou da mensagem, ampliando o alcance dela" sendo uma ação "baseada na percepção [do usuário] de algo como 'interessante' para sua rede social", que legitima e "valoriza a informação que foi originalmente publicada" (RECUERO, 2014, p.120). Os comentários, por outro lado, são percebidos como práticas mais evidentes

\begin{tabular}{|c|c|c|c|c|c|}
\hline (C) RDBCI: Rev. Digit. Bibliotecon. Cienc. Inf. & Campinas, SP & v.16 & n. 2 & p. 365-379 & maio/ago. 2018 \\
\hline
\end{tabular}


do diálogo e não apenas sinalizam a participação, mas trazem uma efetiva contribuição para a conversação, uma vez que compreenderiam uma "participação mais efetiva, demandando um maior esforço e acontecendo quando os usuários têm algo a dizer sobre o assunto" (RECUERO, 2014, p.120).

Assim, considera-se haver um aumento gradativo do comprometimento do usuário com o conteúdo com o qual interage que vai de uma ação do curtir, a outra do compartilhar e por fim, a do comentar. A primeira parece um investimento menor, uma sinalização, a segunda afere maior envolvimento e a última por sua vez indicaria a maior expressão de engajamento. Para cada uma delas é possível pensar sobre a atenção online que a informação científica tem adquirido e o tipo de indicativo aferido para estudos altmétricos. A atenção online é aqui compreendida como aspectos de audiência (alcance) e interações que conteúdos recebem nas mídias sociais (ARAUJO, 2015b).

Para Ringelhan, Wollersheim e Welpe (2015) cientistas e pesquisadores ao usarem o Facebook, por serem indivíduos altamente qualificados, quando "curtem" algo dão visibilidade e audiência a conteúdos relevantes, o que pode ser considerado um indicador valioso do impacto de trabalhos acadêmicos.

Os autores ressaltam a ausência de pesquisas que analisem as interações no Facebook como indicador mais contemporâneo de impacto social de manuscritos científicos, e segundo eles, a literatura sobre altmetria tem revelado uma preferência de análise pelo Twitter. $\mathrm{O}$ "curtir", no entanto, parece ser muito promissor, pois, ao contrário de tweets e outras fontes para dados altmétricos previamente analisadas, ele indica uma ação positiva de gostar de um conteúdo e consequentemente transmiti-lo para sua rede (RINGELHAN; WOLLERSHEIM; WELPE, 2015) o que pode ser estendido às ações de compartilhar e de comentar.

O "compartilhar" pode ser considerado um bom filtro de informação funcionando como ações de recomendações e o "comentar", por sua vez, como um indicativo de receptividade podendo expressar avaliações e debates em torno de artigos recém-publicados. Assim, "artigos bastante compartilhados logo após sua publicação são aqueles de grande apelo para uma área" (IAMARINO, 2013) e os mais "comentados" enfatizam que o apelo tem mobilizado a discussão (BORNMANN, 2014).

Assim, pode-se considerar que as ações de informação expressas nas curtidas, compartilhamentos e comentários no Facebook ajudam a medir a visibilidade nas interações reais e diárias que os usuários desta mídia social (man)têm com pesquisas científicas.

\section{MATERIAL E MÉTODO}

Pesquisa exploratória tendo como universo empírico artigos de periódicos indexados no Repertório da Produção Periódica Brasileira de Ciência da Informação (RPPBCI). Para

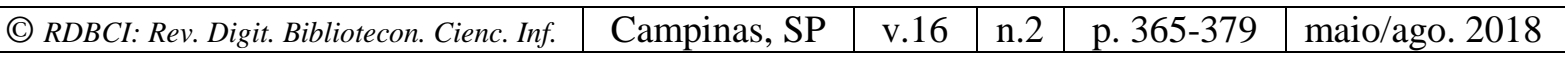


contornar a ausência de um padrão comum de identificação, situação da maioria dos periódicos da área de CI, como Digital Object Indentifier - DOI, a coleta de dados altmétricos do Facebook seguiu as orientações de Araújo e outros (2015) na utilização de uma Application Program Interface (API): < https://developers.facebook.com/tools/explorer/ $>$.

Uma grande vantagem desse tipo de consulta é que diferente do que acontece em serviços de monitoramento de métricas alternativas como o do Altmetric.com, que considera em sua pontuação altmétrica do Facebook apenas postagens em páginas públicas e grupos públicos - desconsiderando curtidas e postagens individuais (ALTMETRIC, 2017) uma busca por URL parametrizada via API, por sua vez, fornece o volume total de todas as interações que esta URL recebe (ALLEN, 2016).

Os metadados das revistas indexadas foram coletados utilizando o protocolo OAIPMH (Open Archives Initiative - Protocol for Metadata Harvesting). O OJS disponibiliza mais de uma URL para cada artigo, sendo a estrutura mais comum uma URL para a exibição dos metadados do artigo e outra uma página com um leitor de PDF incorporado. Porém, há casos de mais de duas URLs, como por exemplo, no caso da revista ser bilíngue.

As URLs são coletadas dos metadados dc:identifier e dc:relation no OAI-PMH. A consulta à API do Facebook (versão 2.9) é feita no momento da exibição do resultado de busca do software e é feita em cada uma das URLs coletadas. Identificamos que a API do Facebook diferencia os resultados das URLs utilizando o prefixo http e https, e devido a esta característica, para cada URL, efetuamos duas consultas na API, uma com cada prefixo. O sistema soma os resultados de todas as URLs e armazena na Banco de Dados não relacional Elasticsearch ${ }^{1}$ os resultados obtidos. Os códigos fonte utilizados estão disponibilizados em: https://github.com/trmurakami/rppbci e a Figura 1 exemplifica o resultado em JSON da API de uma consulta pela URL http://www.revistas.usp.br/incid/article/view/69327.

Figura 1. Resultado da API do Facebook da consulta pela URL

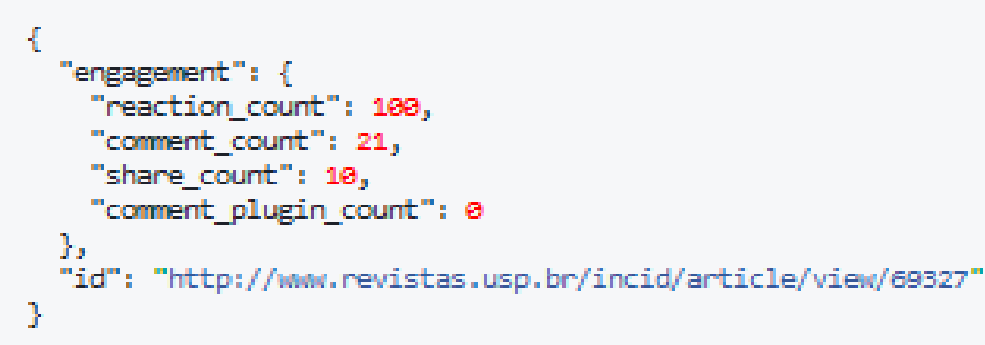

Fonte: dados da pesquisa (2016)

\footnotetext{
${ }^{1}$ Elasticsearch é um mecanismo de serviço de descoberta de pesquisa e análise distribuída, capaz de resolver um número crescente de casos de uso. Disponível em: https://www.elastic.co/products/elasticsearch
}

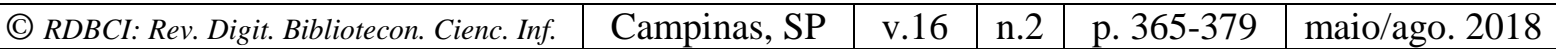


As consultas ocorreram na primeira semana de abril de 2016 e foram parametrizadas pela URL principal e secundária de todos os artigos das 31 revistas presentes no RPPBCI, com sua representação quantitativa em termos de "curtidas" (reaction_count), "compartilhamentos" (share_count) e "comentários" (comment_count). Os dados são apresentados segundo o volume quantitativo por revistas para em seguida discorrer sobre os artigos com maior atenção online.

\section{RESULTADOS E DISCUSSÃo}

Foram analisados os dados de 10.024 artigos publicados pelas 31 revistas das quais 18 $(58,06 \%)$ apresentaram dados altmétricos, os quais somados indicam atenção online de 13.633 interações, sendo no total 8.840 "curtidas" (C1), 2.992 "compartilhamentos" (C2) e 1.801 "comentários" (C3). A Tabela 1 apresenta essa distribuição pela posição (\#) dos periódicos.

Tabela 1. Distribuição dos periódicos por número total de interação no Facebook.

\begin{tabular}{llcccccccc}
\hline$\#$ & Título do periódico & C1 & $\%$ & C2 & $\%$ & C3 & $\%$ & Total & $\%$ \\
\hline 1 & Perspect. Ci. Inf. & 1152 & 13,0 & 422 & 14,1 & 255 & 14,2 & 1829 & 13,4 \\
2 & Rev. Bras. Bibliotec. Doc. & 803 & 9,1 & 208 & 7,0 & 210 & 11,7 & 1221 & 9,0 \\
3 & Encontros Bibli & 767 & 8,7 & 281 & 9,4 & 134 & 7,4 & 1182 & 8,7 \\
4 & Rev. Ib. Americ. Ci. Inf. & 805 & 9,1 & 167 & 5,6 & 107 & 5,9 & 1079 & 7,9 \\
5 & Biblionline & 719 & 8,1 & 144 & 4,8 & 167 & 9,3 & 1030 & 7,6 \\
6 & RECIIS & 562 & 6,4 & 350 & 11,7 & 80 & 4,4 & 992 & 7,3 \\
7 & Revista ACB & 504 & 5,7 & 232 & 7,8 & 138 & 7,7 & 874 & 6,4 \\
8 & Inf. \& Soc.: Estudos & 395 & 4,5 & 214 & 7,2 & 76 & 4,2 & 685 & 5,0 \\
9 & Inf. \& Inf. & 336 & 3,8 & 177 & 5,9 & 77 & 4,3 & 590 & 4,3 \\
10 & Perspect. Gest. Conhec. & 212 & 2,4 & 105 & 3,5 & 76 & 4,2 & 393 & 2,9 \\
11 & PontodeAcesso & 199 & 2,3 & 142 & 4,7 & 50 & 2,8 & 391 & 2,9 \\
12 & Biblos & 225 & 2,5 & 67 & 2,2 & 80 & 4,4 & 372 & 2,7 \\
13 & Ci. Inf. Rev. & 271 & 3,1 & 50 & 1,7 & 51 & 2,8 & 372 & 2,7 \\
14 & Rev. Ci. Inf. & 222 & 2,5 & 87 & 2,9 & 27 & 1,5 & 336 & 2,5 \\
15 & Rev. Bras. Educ. Ci. Inf. & 223 & 2,5 & 42 & 1,4 & 35 & 1,9 & 300 & 2,2 \\
16 & Liinc em Rev. & 213 & 2,4 & 35 & 1,2 & 40 & 2,2 & 288 & 2,1 \\
17 & Rev. Dig. Bibliotec. Ci. Inf. & 177 & 2,0 & 40 & 1,3 & 57 & 3,2 & 274 & 2,0 \\
18 & Transinformacao & 212 & 2,4 & 16 & 0,5 & 45 & 2,5 & 273 & 2,0 \\
19 & InCID & 188 & 2,1 & 40 & 1,3 & 35 & 1,9 & 263 & 1,9 \\
20 & Tend. Pesq. Bras. Ci. Inf. & 178 & 2,0 & 59 & 2,0 & 4 & 0,2 & 241 & 1,8 \\
21 & Inf. \& Tecn.: ITEC & 161 & 1,8 & 55 & 1,8 & 17 & 0,9 & 233 & 1,7
\end{tabular}




\begin{tabular}{|c|c|c|c|c|c|c|c|c|c|}
\hline 22 & Folha de Rosto & 150 & 1,7 & 10 & 0,3 & 20 & 1,1 & 180 & 1,3 \\
\hline 23 & AtoZ: Nov. Prat. Inf. Conhec. & 77 & 0,9 & 13 & 0,4 & 4 & 0,2 & 94 & 0,7 \\
\hline 24 & Braz. Journ. Inf. Sci. & 33 & 0,4 & 4 & 0,1 & 13 & 0,7 & 50 & 0,4 \\
\hline 25 & Informacao@Profissoes & 29 & 0,3 & 6 & 0,2 & 1 & 0,1 & 36 & 0,3 \\
\hline 26 & Pesq. Bras. Ci. Inf. Bibliotec. & 17 & 0,2 & 8 & 0,3 & 2 & 0,1 & 27 & 0,2 \\
\hline 27 & Cad. Inf. Jur. & 8 & 0,1 & 12 & 0,4 & 0 & 0,0 & 20 & 0,1 \\
\hline 28 & EmQuestao & 2 & 0,0 & 4 & 0,1 & 0 & 0,0 & 6 & 0,0 \\
\hline 29 & Bibliot. Univ.: pesq. exp. e persp. & 0 & 0,0 & 2 & 0,1 & 0 & 0,0 & 2 & 0,0 \\
\hline 30 & Biblioteca Escolar em Revista & 0 & 0,0 & 0 & 0,0 & 0 & 0,0 & 0 & 0,0 \\
\hline 31 & IRIS & 0 & 0,0 & 0 & 0,0 & 0 & 0,0 & 0 & 0,0 \\
\hline & Total: & 8840 & 100 & 2992 & 100 & 1801 & 100 & 13633 & 100 \\
\hline & (\%) do total de interações & $\overline{64,8}$ & - & 21,9 & - & 13,2 & - & & \\
\hline
\end{tabular}

Fonte: dados da pesquisa (2016).

Mídias sociais como o Facebook permitem uma comunicação mais fluida e interativa de ideias e têm potencial para levar os periódicos científicos a atingir um número cada vez maior de acessos e leituras, além de possibilitar maior interação entre autores que podem postar comentários sobre o que publicaram em determinadas revistas e responder aos leitores (AMIR et al., 2014).

A revista 'Perspect. Ci. Inf.' alcançou maior audiência na atenção online com total de 1.829 dados de interação $(13,4 \%)$, sendo a única a manter os maiores valores nos três tipos de interação observados. A segunda revista com maior valor total de interação é a 'Rev. Bras. Bibliotec. Doc.' com 1221 (9,0\%), seguida da 'Encontros Bibli' com 1.182 (8,7\%), da 'Rev. Ib. Americ. Ci. Inf.' com 1.079 (7,9\%) e da 'Biblionline' com 1.030 (7,6\%).

No geral, o desempenho das revistas parece não acompanhar ou mesmo não se justificar pelo fato de elas terem ou não uma presença online mantendo perfis em páginas no Facebook. A adesão dos periódicos da área de Biblioteconomia e Ciência da Informação às mídias sociais tem sido tímida de acordo com estudos de Souza et al. (2015) e Costa et al. (2016) e com base nos resultados destas, das 10 revistas com melhor posição no ranking da Tabela 1, apenas a RECIIS e a Revista ACB possuem página no Facebook. O que nos faz inferir que os dados altmétricos são gerados pelos autores dos artigos, sua rede de contatos e a comunidade leitora e interessada nos assuntos que os artigos tratam.

Ainda que na maioria dos casos os valores dos "comentários", sejam menores que as "curtidas" e "compartilhamentos", algumas revistas, mesmo bem pontuadas em um deles, descem no posicionamento em função dos outros. A 'RECIIS', por exemplo, embora seja a sexta do ranking geral, com 992 (7,3\%) interações apresenta o segundo maior valor de interações de compartilhamento. Outra questão a ser observada é que em alguns casos, poucos

\begin{tabular}{l|l|l|l|l|l}
\hline (C) RDBCI: Rev. Digit. Bibliotecon. Cienc. Inf. & Campinas, SP & v.16 & n.2 & p. 365-379 & maio/ago. 2018 \\
\hline
\end{tabular}


artigos são responsáveis pelo bom desempenho do periódico em um dos tipos de interação.

As ações de "curtir" representam o tipo de interação mais evidente do universo analisado e correspondem a 64,8\% das interações, seguida do "compartilhar" com $21,9 \%$. De acordo com Recuero (2014, p.121) "o objetivo do uso de ferramentas como o curtir e o compartilhar é aquele, justamente, de legitimar o que está sendo dito, concordando ou assertindo" e costuma ser associado a uma ação positiva em relação ao conteúdo.

A ação de "comentar" é menos expressiva que as demais, com 13,2\%. O "comentar", por demandar maior comprometimento na possível conversação costuma ser menos frequente (RECUERO, 2014) o que equivale dizer que as possíveis discussões geradas em torno dos artigos ainda é relativamente baixa.

Embora medidas tradicionais da reputação de um periódico científico, como o fator de impacto ainda sejam (e continuarão sendo) amplamente utilizadas, é impossível ignorar o papel de mídias sociais como o Facebook como fonte de dados altmétricos e o seu "impacto social" na atenção online combinada das interações de curtir, comentar e compartilhar que as revistas recebem. Pesquisadores como Karimkhani, Gamble e Dellavalle (2014) chegam a dizer que nessa nova direção que busca impacto acadêmico e social, os autores que avaliem rotineiramente os fatores de impacto do periódico no intuito de determinar para qual revista enviar seus manuscritos, também podem passar a considerar o impacto das mídias sociais dessas revistas.

Para uma melhor aproximação do universo analisado, tendo em vista o volume de artigos, foi realizado um recorte com os dez mais expressivos, ou seja, um ranking com os artigos que obtiveram os maiores índices altmétricos segundo suas interações no Facebook.

Tais artigos podem ser visualizados na Tabela 2 com descrição do título, seus autores, título do periódico, ano de publicação e o número de curtidas $(\mathrm{C} 1)$, compartilhamentos $(\mathrm{C} 2)$, comentários (C3) e a soma deles.

Tabela 2. Dez artigos que obtiveram mais atenção online

\begin{tabular}{llllllll}
\hline Título & Autores & Periódico & Ano & C1 & C2 & C3 & Total \\
\hline $\begin{array}{l}\text { O bibliotecário na gestão de } \\
\text { pessoas: um estudo baseado na } \\
\text { gestão do banco de dados do jornal } \\
\text { o povo }\end{array}$ & $\begin{array}{l}\text { Pires Santos, F. E.; } \\
\text { Silva, A. K. P. da; } \\
\text { Ayres, M. T. L. }\end{array}$ & Biblionline & 2014 & 145 & 2 & 38 & $\mathbf{1 8 5}$ \\
$\begin{array}{l}\text { A digitalização de documentos } \\
\text { arquivísticos no contexto brasileiro }\end{array}$ & $\begin{array}{l}\text { Schäfer, M. B.; } \\
\text { Flores, D. }\end{array}$ & $\begin{array}{l}\text { Tend. da } \\
\text { Pesq. Bras. } 2013 \\
\text { em Ci.Inf. }\end{array}$ & 146 & 1 & 34 & $\mathbf{1 8 4}$
\end{tabular}




\begin{tabular}{|c|c|c|c|c|c|c|c|}
\hline $\begin{array}{l}\text { A brief history of academic libraries } \\
\text { automation in Brazil and some } \\
\text { future perspectives }\end{array}$ & $\begin{array}{l}\text { Viana, M.; } \\
\text { Mazzardo, M. }\end{array}$ & $\begin{array}{l}\text { Rev. Ib. } \\
\text { Americ. } \\
\text { Ci. Inf. }\end{array}$ & 2015 & 125 & 29 & 24 & 178 \\
\hline $\begin{array}{l}\text { Usuário, não! Interagente. Proposta } \\
\text { de um novo termo para um novo } \\
\text { tempo }\end{array}$ & Corrêa, E. C. D. & $\begin{array}{l}\text { Encontros } \\
\text { Bibli }\end{array}$ & 2014 & 100 & 31 & 28 & 159 \\
\hline $\begin{array}{l}\text { History of paper: evolutionary } \\
\text { panorama of production techniques } \\
\text { and the implications for its } \\
\text { preservation }\end{array}$ & $\begin{array}{l}\text { Fritoli, C. L.; Krüger, } \\
\text { E. L.; Carvalho, S. } \\
\text { K. de P. }\end{array}$ & $\begin{array}{l}\text { Rev. Ib. } \\
\text { Americ. } \\
\text { Ci. Inf. }\end{array}$ & 2016 & 93 & 18 & 46 & 157 \\
\hline $\begin{array}{l}\text { Políticas de preservação digital para } \\
\text { documentos arquivísticos }\end{array}$ & $\begin{array}{l}\text { Santos, H. M. dos; } \\
\text { Flores, D. }\end{array}$ & $\begin{array}{l}\text { Perspect. } \\
\text { Ci. Inf. }\end{array}$ & 2015 & 114 & 15 & 22 & 151 \\
\hline $\begin{array}{l}\text { Use of social network to support } \\
\text { visually impaired people: A } \\
\text { Facebook case study }\end{array}$ & $\begin{array}{l}\text { Caran, G. M.; } \\
\text { Santini, R. M.; } \\
\text { Biolchini, J. C. de A. }\end{array}$ & Transinf. & 2016 & 109 & 37 & 2 & 148 \\
\hline $\begin{array}{l}\text { The role of archives of the federal } \\
\text { institutions of higher education and } \\
\text { the experience of the Central } \\
\text { Archive of the University of Brasilia }\end{array}$ & Roncaglio, C. & $\begin{array}{l}\text { Rev. Ib. } \\
\text { Americ. } \\
\text { Ci. Inf. }\end{array}$ & 2015 & 104 & 9 & 24 & 137 \\
\hline $\begin{array}{l}\text { Memórias em rede: as fotografias } \\
\text { em ambientes virtuais }\end{array}$ & $\begin{array}{l}\text { Morigi, V. J.; } \\
\text { Massoni, L. F. H. }\end{array}$ & $\begin{array}{l}\text { Liinc em } \\
\text { Revista }\end{array}$ & 2015 & 107 & 23 & 6 & 136 \\
\hline $\begin{array}{l}\text { The evolution of the topic of } \\
\text { Information literacy in Brazil: a } \\
\text { bibliographic study from } 2006 \text { to } \\
2013\end{array}$ & $\begin{array}{l}\text { Trein, J. M.; } \\
\text { Vitorino, E. V. }\end{array}$ & $\begin{array}{l}\text { Rev. Bras. } \\
\text { Bibliotec. } \\
\text { Doc. }\end{array}$ & 2016 & 99 & 24 & 10 & 133 \\
\hline
\end{tabular}

\footnotetext{
Fonte: Dados da pesquisa.
}

Conforme esperado de indicadores altmétricos, artigos mais recentes tendem a obter maior atenção online, e consequentemente uma pontuação elevada (ARAUJO, 2015a; BARROS, 2015). Os artigos apresentados na tabela foram publicados nos anos de 2013 (1) 2014 (2), 2015 (4) e 2016 (3). O artigo 'O bibliotecário na gestão de pessoas: um estudo baseado na gestão do banco de dados do jornal o povo' de autoria de Pires Santos, Silva e Ayres (2014) é o que recebeu mais atenção online com total de 185 interações

Assim como no ranking dos periódicos, mais uma vez os valores das interações de "curtidas" superam os de "compartilhamento" e "comentários", mas alguns artigos, mesmo bem pontuados em um tipo de interação, descem no posicionamento em função de outras.

O artigo 'A digitalização de documentos arquivísticos no contexto brasileiro' de Schäfer e Flores (2013), por exemplo, ocupa o segundo lugar nas interações totais, mas é o primeiro em 'curtidas' (146, uma curtida acima do primeiro lugar geral). O artigo 'Use of social network to support visually impaired people: A Facebook case study’ de Caran, Santini e Biolchini (2016),

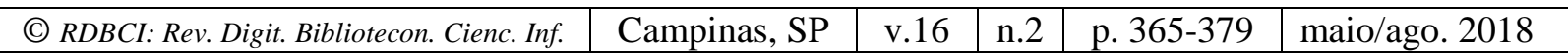


embora ocupe a sétima posição no total de interações é o mais disseminado, contando com 37 compartilhamentos. E, por fim, o artigo 'History of paper: evolutionary panorama of production techniques and the implications for its preservation' de Fritoli, Krüger e Carvalho (2016), mesmo ocupando a quinta posição geral, é o que mais engajou usuários para conversações, com 46 comentários.

\section{CONSIDERAÇÕES FINAIS}

Interações com conteúdo de artigos científicos no Facebook são ricas fontes de dados altmétricos e merecem maior atenção por parte de estudiosos desse campo, elas podem ser benéficas como uma métrica agregada porque fornecem uma visão adicional sobre o desempenho científico dos artigos quando curtidos, compartilhados ou comentados.

O estudo realizado oferece subsídios teórico-metodológicos para outras pesquisas interessadas no potencial da atenção online que artigos científicos recebem no Facebook, seja pelas reflexões sobre as especificidades desta mídia social para a comunicação científica, quanto no ganho prático no método de coleta de dados via API que não só contorna o problema de ausência do DOI de algumas revistas, mas também cobre um espectro maior da atenção online por considerar na busca dados de conversas privadas. Em relação ao DOI torna-se necessário estudar formas de agregar dados das revistas que possuem o identificador para que a atenção online seja completa.

Sobre esta última questão, vale lembrar que dados de interações públicas do Facebook, comumente fornecidos em algumas plataformas como o Altmetric.com, representam uma pequena parcela do engajamento que uma pesquisa científica gera nesta mídia social, ou seja, são apenas uma fração do envolvimento total do Facebook, não sendo uma métrica suficiente e confiável para entender o escopo do engajamento.

De acordo com Allen (2016) nas interações privadas são onde as conversações reais acontecem. Para o autor, são nelas que ocorrem as conversas entre indivíduos sobre o que importa para eles e, portanto, medir as conversas mais pessoais do Facebook pode fornecer uma indicação digna de promoção e atenção online que as pesquisas recebem. E para fins de questões éticas de pesquisa, é importante ressaltar que este recurso de coleta via API está em conformidade com os Termos de Serviço de privacidade do Facebook e o mesmo não divulga a identidade de provedores de comentários, preservando a privacidade dos mesmos.

No total de periódicos analisados apenas duas revistas não apresentaram dados altmétricos quando consultadas tanto pela URL principal quanto pela secundária, são elas: 'Biblioteca Escolar em Revista' e 'IRIS - Revista de Informação, Memória e Tecnologia'.

Para continuidade da pesquisa e melhor compreensão do que são e o que representam, os dados serão analisados no seu conjunto para saber se há algum padrão de comportamento no

\begin{tabular}{|c|c|c|c|c|c|}
\hline (C) RDBCI: Rev. Digit. Bibliotecon. Cienc. Inf. & Campinas, SP & v.16 & n. 2 & p. 365-379 & maio/ago. 2018 \\
\hline
\end{tabular}


que tange aos valores gerais e específicos de interação e se, por exemplo, uma categorização temática pode indicar maior ou menor grau de atenção online que os artigos recebem no Facebook.

\section{REFERÊNCIAS}

ALLEN, Polly. PlumX's Facebook Altmetrics - Measure Up! Plum Analytics., Ago., 2016. Disponível em: < http://plumanalytics.com/plumx-facebook-altmetrics-measure-up/ >. Acesso em 13 dez., 2016.

AMIR, M. et al. Social Networking Sites Emerging and Essential Tools for Communication in Dermatology. JAMA Dermatol. v.150, n.1, p.56-60, 2014. Disponível em: <http://dx.doi.org/doi:10.1001/jamadermatol.2013.6340>. Acesso em 19 dez., 2017.

ARAUJO, Ronaldo Ferreira. Mídias sociais e comunicação científica: análise altmétrica em artigos de periódicos da ciência da informação. Em Questão, v. 21, p.96-109, 2015 a. Disponível em: <http://dx.doi.org/10.19132/1808-5245211.96-109 >. Acesso em 24 jan., 2017.

ARAUJO, Ronaldo Ferreira. Marketing científico digital e métricas alternativas para periódicos: da visibilidade ao engajamento. Perspectivas em Ciência da Informação, v. 20, n.3, p. 67-84, 2015b. Disponível em: <http://dx.doi.org/10.1590/1981-5344/2402>. Acesso em 03 ago., 2016.

ARAUJO, Ronaldo Ferreira; MURAKAMI, Tiago Rodrigo Marçal; LARA, Jan Leduc de; FAUSTO, Sibele. Does the Global South Have Altmetrics? Analyzing a Brazilian LIS Journal. In: International Society of Scientometrics and Informetrics Conference., Istambul, Turquia, v. 15. p. 111-112. 2015, Istambul, Turquia.. Proceedings... Bogaziçi University Printhouse. Istambul: SALAH, A. A.; TONTA, Y.; AKDAG SALAH, A. A.; SUGIMOTO, C. et al. (Eds.), 2015.

BARROS Moreno. A.. Altmetrics: métricas alternativas de impacto científico com base em redes sociais. Perspectivas em Ciência da Informação, v.20, n.2, p.19-37, abr./jun. 2015. Disponível em: <http://portaldeperiodicos.eci.ufmg.br/index.php/pci/article/view/1782>. Acesso em 11 nov., 2016.

BARTH, Mauricio; PACHECO, Jeison; PINHEIRO, Cristiano Max Pereira. Interação, engajamento e crowdsourcing: um estudo do caso The Johnny Cash Project. Comun. \& Inf., Goiânia, v. 18, n. 1, p.76-96, jan./jun. 2015. Disponível em:

<https://www.revistas.ufg.br/ci/article/view/33738>. Acesso em 17 set., 2016.

BLATTMANN, Ursula.; SANTOS, Raimundo Nonato Macedo dos. Revistas científicas brasileiras e sua visibilidade no acesso aberto. Informação \& Sociedade (UFPB. Online), v. 24, p. 99-106, 2014. Disponível em: < http://www.ies.ufpb.br/ojs/index.php/ies/article/view/21807>. Acesso em 23 mar., 2016. 
BOON, Chia Yew; FOON, Joan Wee Jee. Altmetrics is an indication of quality research or just hot topics. In: IATUL Conferences, 35th. Purdue University. Espoo, Filand, 2014.

Proceedings... Espoo, Filand, 2014. Disponível em: <

http://docs.lib.purdue.edu/cgi/viewcontent.cgi?article=2034\&context=iatul >. Acesso em 07 set., 2016.

BORNMANN, Lutz. Do altmetrics point to the broader impact of research? An overview of benefits and disadvantages of altmetrics. Journal of Informetrics, v.8, n.4, p.895-903, out., 2014.

CARAN, Gustavo Miranda; SANTINI, Rose Marie; BIOLCHINI, Jorge Calmon de Almeida. Use of social network to support visually impaired people: A Facebook case study.

Transinformação, Campinas, v. 28, n. 2, p. 173-180, ago., 2016. Disponível em: < http://dx.doi.org/10.1590/2318-08892016000200004 >. Acesso em 20 ago., 2016.

COSTA, Luciana Ferreira. et al. O uso de mídias sociais por revistas científicas da área da ciência da informação para ações de marketing digital. Revista ACB: Biblioteconomia em Santa Catarina, Florianópolis, v. 21, n. 2, p. 338-358, abr./ jul., 2016. Disponível em: < https://revista.acbsc.org.br/racb/article/view/1159>. Acesso em 20 dez., 2017.

FRITOLI, Clara Landim; KRÜGER, Eduardo; CARVALHO, Silmara Küster de Paula.. History of paper: evolutionary panorama of production techniques and the implications for its preservation. RICI: R.Ibero-amer. Ci. Inf., Brasília, v. 9, n. 2, p. 475-502, jul./ dez. 2016. Disponível em: < http://periodicos.unb.br/index.php/RICI/article/view/18630 > . Acesso em 20 ago., 2016.

GOUVEIA, Fábio Castro. A altmetria e a interface entre a ciência e a sociedade. Trab. Educ. Saúde, Rio de Janeiro, v. 14 n. 3, p. 643-651,set./dez. 2016. Disponível em: < http://dx.doi.org/10.1590/1981-7746-sip00126 >. Acesso em 20 ago., 2016.

GOUVEIA, Fábio Castro. Altmetria: métricas de produção científica para além das citações. Liinc em Revista, Rio de Janeiro, v. 9, n. 1, p. 214-227, mai., 2013. Disponível em: < https://doi.org/10.18617/liinc.v9i1.569>. Acesso em 13 jul., 2016.

HAUSTEIN, Stefanie; BOWMAN, Timothy D.; MACALUSO, Benoit; SUGIMOTO, Cassidy R.; LARIVIÈRE, Vincent; BÖRNER, Katy.. Measuring Twitter activity of arXiv eprints and published papers. In: Altmetrics14: expanding impacts and metrics. An ACM Web Science Conference 2014 Workshop. Bloomington, IN. Disponível em:

<https://doi.org/10.6084/m9.figshare.1041514.v1 >. Acesso em: 13 ago., 2016.

HOLMBERG, K. et al. Astrophysicists' Conversational Connections on Twitter. PLoS ONE, v.9, n.8, ago., 2014. Disponível em: < https://doi.org/10.1371/journal.pone.0106086 >. Acesso em 04 set., 2016.

IAMARINO, Átila. Você compartilha, eu curto e nós geramos métricas. SciELO em Perspectiva. Ago., 2013. Disponível em: < http://blog.scielo.org/blog/2013/08/08/vocecompartilha-eu-curto-e-nos-geramos-metricas/ > . Acesso em 20 abr., 2015. 
KARIMKHANI, C.; GAMBLE, R.; DELLAVALLE, R. P. Social media impact factor: the top ten dermatology journals on facebook and twitter. Dermatol Online J. v.20, n.4, abr., p.223-227, 2014. Disponível em: < https://escholarship.org/uc/item/4rb3w037>. Acesso em 21 dez., 2017.

PIRES SANTOS; F. E.; SILVA, A. K. P.; AYRES, A. T. L.. O bibliotecário na gestão de pessoas: um estudo baseado na gestão do banco de dados do jornal o povo. Biblionline, João Pessoa, v. 10, n. 2, p. 138-153, 2014. Disponível em:

$<$ http://periodicos.ufpb.br/ojs2/index.php/biblio/article/view/15965 > . Acesso em 20 ago., 2016.

PRIEM, Jason; GROTH, P.; TARABORELLI, D. The altmetrics collection. PLOS ONE. v.7, n.11, nov., 2012. doi: 10.1371/journal.pone.0048753.pmid:23133655. Disponível em: < http://journals.plos.org/plosone/article?id=10.1371/journal.pone.0048753 >. Acesso em 13 ago., 2014.

RECUERO, Raquel. Curtir, compartilhar, comentar: trabalho de face, conversação e redes sociais no Facebook. Verso e Reverso, v.28, n.68, p.114-124, mai. Ago., 2014. Disponível em: <http://dx.doi.org/10.4013/ver.2014.28.68.06>. Acesso em 23 ago., 2015.

RINGELHAN, S.; WOLLERSHEIM, J.; WELPE, I. M. I Like, I Cite? Do Facebook Likes Predict the Impact of Scientific Work? PLoS ONE, v.10, n.8, ago., 2015. Disponível em: <http://dx.doi.org/doi:10.1371/journal.pone.0134389>. Acesso em 7 dez., 2016

SCHNITZLER, Katy; DAVIES, Nigel; ROSS, Fiona; HARRIS, Ruth. Using Twitter ${ }^{\text {TM }}$ to drive research impact: A discussion of strategies, opportunities and challenges. International Journal of Nursing Studies, v.59, p.15-26, jul., 2016. Disponível em: <

http://dx.doi.org/10.1016/j.ijnurstu.2016.02.004 >. Acesso em 13 dez., 2016.

SCHÄFER, Murilo Billig; FLORES, Daniel. A digitalização de documentos arquivísticos no contexto brasileiro. Tendências da Pesquisa Brasileira em Ciência da Informação, v.6, n.2, jul./dez.2013. Disponível em: < http://inseer.ibict.br/ancib/index.php/tpbci/article/view/116/158> . Acesso em 20 ago., 2016.

SHEMA, Hadas; BAR-ILAN, Judit; THELWALL, Mike. Do blog citations correlate with a higher number of future citations? Research blogs as a potential source for alternative metrics. Journal of the Association for Information Science and Technology. v.65, n.5, p.1018-1027, jan., 2014. Disponível em: <https://doi.org/10.1002/asi.23037>. >. Acesso em 24 jul., 2016.

SOUZA, Uarlens de Jesus. et al. O uso das redes sociais pelos periódicos brasileiros de biblioteconomia e ciência da informação. Revista ACB: Biblioteconomia em Santa Catarina, Florianópolis, v. 20, n. 3, p. 584-591, set./dez., 2015. Disponível em: <https://revista.acbsc.org.br/racb/article/view/1101>. Acesso em 20 dez., 2017.

THELWALL, Mike; HAUSTEIN, Stefanie; LARIVIÈRE, Vincent; SUGIMOTO, Cassidy R.. Do Altmetrics Work? Twitter and Ten Other Social Web Services. PLoS ONE, v.8, n.5, 
mai., 2013. Disponível em: < https://doi.org/10.1371/journal.pone.0064841 >. Acesso em 21 nov., 2016.

VANTI, Nadia; SANZ-CASADO, Elias. Altmetria: a métrica social a serviço de uma ciência mais democrática. Transinformação, Campinas, v. 28, n. 3, p. 349-358, dez., 2016.

Disponível em: < http://periodicos.puc-

campinas.edu.br/seer/index.php/transinfo/article/view/2644>. Acesso em 11 jan., 2017.

WINTER, Joost C. F.. The relationship between tweets, citations, and article views for PLOS ONE articles. Scientometrics, v.102, n.2, p.1773-1779, fev., 2015. Disponível em:

<https://doi.org/10.1007/s11192-014-1445-x >. Acesso em 02 jan., 2017.
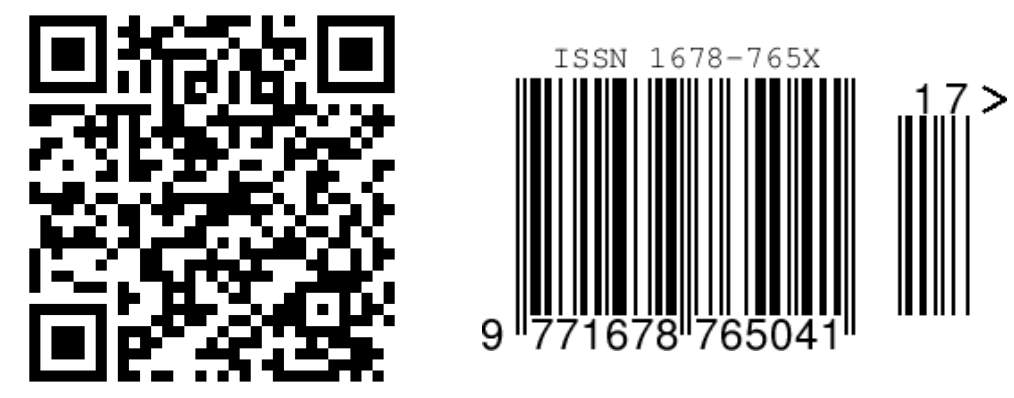